\title{
Percepção das adolescentes sobre a consulta de Enfermagem na Atenção Básica de Saúde
}

\author{
Adolescent's perception about nursing consultation in Primary Health Care
}

Percepción de los adolescentes a cerca de la consulta de enfermería en Atención Primaria de Salud

Flávia Regina da Costa Abreu ${ }^{1 *}$, Letícia Hayanne de Oliveira Galvãoㄹ, Ruthyally Kelly de Moraes Sobral ${ }^{1}$, Wanessa Barros da Silva ${ }^{1}$, Júlio César Bernardino da Silva ${ }^{1}$, Alecsandra Gomes de Lucena Oliveira $^{1}$, Weslla Karla Albuquerque Silva de Paula², Vanessa Juvino de Sousa'1.

\section{RESUMO}

Objetivo: Descrever a percepção de adolescentes sobre a consulta de Enfermagem na Atenção Básica de Saúde. Métodos: Estudo descritivo exploratório, com abordagem qualitativa, realizado em uma Unidade Básica de Saúde de um município pernambucano. Entrevistou-se 10 adolescentes do sexo feminino. A coleta de dados foi realizada a partir de um roteiro de entrevista semiestruturada. A análise de conteúdo temática, proposto por Bardin foi utilizada para a análise dos dados, onde emergiram categorias temáticas. O estudo foi aprovado por Comitê de Ética em Pesquisa. Resultados: As adolescentes consideram a consulta de Enfermagem como um importante meio de esclarecimentos e orientações de saúde, permitindo a criação de um vínculo de confiança entre o Enfermeiro e a adolescente. Entretanto, identificou-se uma carência de atividades e de ações internas e externas no contexto da Atenção Básica para esse público alvo. Conclusão: Ressalta-se a necessidade de mais estudos relacionados a esse tema, com o objetivo de cada vez mais compreender as necessidades das adolescentes e encontrar métodos eficazes que possibilitem modificar o cenário da saúde do adolescente no Brasil.

Palavras-chave: Atenção primária à saúde, Processo de enfermagem, Desenvolvimento do adolescente.

\begin{abstract}
Objective: Describe the perception of adolescents about the consultation of Nursing in Primary Health Care. Methods: Descriptive exploratory study, with a qualitative approach, carried out in a Basic Health Unit in a municipality in Pernambuco. 10 female adolescents were interviewed. Data collection was performed from a semi-structured interview script. Thematic content analysis proposed by Bardin was used for data analysis, where thematic categories emerged. The study was approved by the Research Ethics Committee. Results: The adolescents consider the nursing consultation as an important means of clarification and health guidelines, allowing the creation of a bond of trust between the nurse and the adolescent. However, a lack of internal and external activities and actions was identified in the context of Primary Care for this target audience. Conclusion: It is emphasized the need for further studies related to this theme, with the objective of increasingly understanding the needs of adolescents and finding effective methods that allow modifying the scenario of adolescent health in Brazil.
\end{abstract}

Key words: Primary health care, Nursing process, Adolescent development.

\section{RESUMEN}

Objetivo: Describer la percepción de los adolescentes sobre la consulta de Enfermería en la Atención Primaria de Salud. Métodos: Estudio exploratorio descriptivo, con enfoque cualitativo, realizado en una Unidad Básica de Salud en un municipio de Pernambuco. 10 mujeres adolescentes fueron entrevistadas. La recolección de datos se realizó a partir de un guión de entrevista semiestructurada. El análisis de contenido

${ }^{1}$ Centro Universitário Tabosa de Almeida (ASCES-UNITA), Caruaru - PE.

*E-mail: flavia.regina.abreu@gmail.com

2 Universidade Federal de Pernambuco (UFPE), Recife - PE.

SUBMTIDO EM: 1/2020

ACEITO EM: 2/2020

PUBLICADO EM: $3 / 2020$ 
temático propuesto por Bardin se utilizó para el análisis de datos, donde surgieron categorías temáticas. El estudio fue aprobado por el Comité de Ética en Investigación. Resultados: Los adolescentes consideran la consulta de enfermería como un medio importante de aclaración y pautas de salud, permitiendo la creación de un vínculo de confianza entre la enfermera y el adolescente. Sin embargo, se identificó una falta de actividades y acciones internas y externas en el contexto de Atención Primaria para este público objetivo. Conclusión: Hacemos hincapié en la necesidad de realizar más estudios relacionados con este tema, con el objetivo de comprender cada vez más las necesidades de los adolescentes y encontrar métodos para modificar el escenario de la salud de los adolescentes en Brasil.

Palabras clave: Atención primaria de salud, Proceso de enfermería, Desarrollo adolescente.

\section{INTRODUÇÃO}

Conceitua-se a Atenção Básica $(\mathrm{AB})$ como um dos pilares mais importantes do Sistema Único de Saúde (SUS), e a porta preferencial de entrada do serviço de saúde. Caracteriza-se como um conjunto de ações individuais ou coletiva que tem como princípios a promoção e proteção da saúde, prevenção de agravos, o diagnóstico, o tratamento e a reabilitação de danos, visando a uma assistência que gere benefícios e autonomia para a população impactando nos determinantes e condicionantes da saúde, através de atividades assistenciais e educativas (BRASIL, 2011).

Compreende-se que a $A B$ desempenha algumas funções a fim de contribuir com o funcionamento das Redes de Atenção à Saúde, como: ser base, ser resolutiva, coordenar o cuidado e ordenar a rede. Ressalvase o objetivo de identificar as necessidades, demandas e riscos da população sob sua responsabilidade, bem como gerenciando projetos terapêuticos singulares capazes de atender as carências da comunidade (BRASIL, 2011).

Nesse sentido, o profissional enfermeiro necessita realizar um trabalho que o possibilite identificar as principais demandas de sua área adscrita, de modo que alcance em suas ações todos os usuários, entretanto, deve-se reconhecer a dificuldade da $A B$ em conseguir abranger alguns grupos específicos, como os adolescentes. Identifica-se uma resistência por parte desse grupo em compreender a necessidade da utilização de métodos preventivos de agravos à saúde, a base da atenção primária (VIEIRA RP, et al, 2014).

Identifica-se a necessidade de encontrar métodos que atraíam os adolescentes e jovens para a $A B$, com a finalidade de trabalhar educação em saúde, gerando informação para esse público que se encontra em fase de transição entre a infância e a vida adulta, período esse que compreende uma série de transformações sociais, culturais, emocionais, hormonais, corporais, entre outras, gerando dúvidas e questionamentos (QUEIROZ MVO, et al, 2011).

Ressalta-se, o número crescente de adolescentes grávidas, fato que deixa evidente o início de uma vida sexual precoce, bem como a percepção de que o uso de métodos preventivos é negligenciado, gerando um aumento da susceptibilidade dessas meninas a doenças sexualmente transmissíveis, assim, a presença das adolescentes no cenário da atenção básica de saúde proporciona melhorias na qualidade de vida desse público (BRASIL, 2010).

Entende-se, portanto, que a consulta de enfermagem na Atenção Básica para meninas adolescentes proporciona esclarecimentos sobre a saúde e as várias mudanças vivenciadas por esse público, uma vez que é possível realizar orientações acerca de hábitos alimentares, prática de exercício físico, ciclo menstrual, sexualidade, transformações corporais, entre outros, essa uma ação capaz de promover e prevenir agravos à saúde das adolescentes (FERRARI RAP, et al, 2006).

Desse modo, ressalta-se a importância do profissional de Enfermagem em atuar no contexto da assistência primária à saúde de modo a aproximar os adolescentes e inseri-los numa rede de cuidados, visto que uma consulta de Enfermagem sistematizada e com uma visão ampla possibilita uma análise global do processo saúde-adoecimento dessa população específica. Portanto, o objetivo desse estudo foi de descrever a percepção de adolescentes sobre a consulta de enfermagem na atenção básica de saúde. 


\section{MÉTODOS}

Trata-se de um estudo qualitativo, do tipo descritivo, realizado no período de maio a julho de 2017 , com meninas adolescentes de 15 a 19 anos, totalizando 10 (dez) amostras. Selecionaram-se as participantes a partir dos critérios de inclusão: adolescentes do sexo feminino entre a faixa etária de 15 a 19 anos, cadastradas em uma Unidade Básica de Saúde (UBS) da zona urbana, escolhida por conveniência, de um município Pernambucano. Excluíram-se da pesquisa as adolescentes grávidas ou com incapacidade cognitiva/transtornos psicológicos que dificultasse o processo de entrevista.

Coletaram-se os dados por meio de entrevistas presenciais e individuais, a partir das adolescentes cadastradas na UBS, por meio de um roteiro semiestruturado, composto por questões norteadoras direcionadas aos objetivos do estudo. Tais questões foram: 1) Para você como foi à consulta de enfermagem? 2) Quais os sentimentos vivenciados durante a consulta de enfermagem? 3) Você acredita que o atendimento prestado a você influenciará em alguma mudança de comportamento? 4) Com qual frequência você desejaria ter essa consulta de enfermagem?

O processo de coleta de dados aconteceu a partir de duas fases. A princípio, foi agendado, mediante a aceitação das participantes, a realização de uma consulta de Enfermagem, e posteriormente as consultas, a segunda etapa se deu através da realização das entrevistas, que foram gravadas em mídia digital e realizadas em um ambiente reservado e tranquilo na própria UBS, a fim de não se alterar a dinâmica do serviço e garantir a privacidade. Delimitou-se o número dos participantes pela saturação dos dados obtidos.

Gravaram-se e transcreveram-se as falas obtidas, a partir das entrevistas realizadas, analisando-as, posteriormente com a Análise de Conteúdo na modalidade temática proposta por Bardin L (2011), a qual se caracteriza como um conjunto de técnicas de análise utilizando procedimentos sistemáticos e objetivos de descrição de conteúdo dos discursos. Identifica-se nos resultados, as adolescentes pela letra "A" e enumeradas de acordo com a sequência da realização da entrevista, preservando assim suas identidades.

O presente artigo foi enviado ao Comitê de Ética em Pesquisa (CEP), aprovando-o em setembro de 2017 (CAAE: 62276316.3.0000.5203/Parecer: 1.912.729). Coletaram-se os dados somente após a participação voluntária através da assinatura do Termo de Assentimento Livre e Esclarecido, sendo autorizada a participar pelo seu responsável legal através do Termo de Consentimento Livre e Esclarecido, sendo-Ihes assegurados, o sigilo e o anonimato das informações, de acordo com as recomendações preconizadas na Resolução 466/12 e 510/16 do Conselho Nacional de Saúde, em que se respeitam os preceitos éticos tanto na aquisição quanto na divulgação dos dados (BRASIL 2016; BRASIL, 2017).

\section{RESULTADOS E DISCUSSÃO}

Para caracterização das entrevistadas, detalha-se que, das 10 adolescentes, quatro possuíam 15 anos de idade (40\%), quatro com 16 anos (40\%), e duas com 17 e 18 anos respectivamente. Em relação à escolaridade, $100 \%$ possuíam o grau de ensino médio incompleto; ao questionar sua religião, cinco adolescentes declararam ser evangélica (50\%), quatro de religião católica $(40 \%)$ e uma sem religião (10\%); ainda, investigou-se a raça das participantes, sendo oito auto declaradas como parda (80\%) e duas como branca (20\%).

Por meio da análise temática dos dados, após a transcrição das entrevistas, elencaram-se três categorias temáticas: "A consulta de enfermagem possibilita esclarecer dúvidas das adolescentes"; "A criação do vínculo de confiança através da consulta"; "Falta de atividades educativas em saúde na UBS".

\section{A consulta de enfermagem possibilita esclarecer dúvidas das adolescentes}

As adolescentes consideram a consulta de enfermagem como importante meio de esclarecimentos e orientações de saúde durante o período da adolescência.

"E algo novo pra mim, eu nunca procurei o posto de saúde muito, pra conversar, tirar alguma dúvida [...] dúvidas sobre meu corpo e sobre o que acontece com as meninas na adolescência." (A1) 
"Ah eu gostei. Eu achei legal. [...] Mas eu não fiquei com vergonha e falei tudo que ela perguntava, e eu gostei." (A3)

"A foi interessante, porque [...] o que eu não sabia fiquei sabendo. E assim, a pessoa "tira" algumas dúvidas, que a pessoa precisa, porque aí eu acho que, sei lá, o pessoal aí, não, não dá atenção pros adolescentes." (A5)

"Medo. (Risos) [...] Porque eu pensava que iam dar uma injeção em mim. (Risos) Essa consulta ensina coisas, coisas que a pessoa pode fazer certo. Que foi bom demais." (A9)

Identifica-se através das falas a satisfação das adolescentes ao vivenciar a consulta de enfermagem e ter a oportunidade de tirar dúvidas sobre hábitos saudáveis e mudanças corporais na adolescência, bem como realizar questionamentos sobre sexo e sexualidade. Além disso, as entrevistadas demonstram em suas falas a quebra do estigma social da consulta de enfermagem ser apenas procedimentos invasivos e curativos.

Esse é um tema por vezes bastante banalizado, considerado por educadores como transversal. Seria necessário que não só profissionais de saúde buscassem conversar e esclarecer dúvidas, mas também que essa discussão acontecesse no ambiente escolar e familiar. Entendendo que para trabalhar com adolescentes deve-se ter o cuidado de como falar e agir, principalmente sobre temas relacionados ao seu corpo por gerar timidez. Por esse motivo é importante respeitar a privacidade e individualidade de cada uma (ALENCAR RA, et al, 2008).

É perceptível que o atendimento individualizado deixa as adolescentes mais tranquilas e seguras para realizar suas indagações, referir queixas e pedir aconselhamentos. Grosman, et al (2004), afirmam que a consulta de enfermagem deve compreender a suscetibilidade desse grupo, identificando suas necessidades, e não deixando de considerar a complexidade das problemáticas que as cercam.

O atendimento de enfermagem deverá influenciar essas meninas a compreender a importância do cuidado com sua saúde, bem como torná-las empoderadas, ou seja, capazes de ter autonomia sobre sua vida e suas ações, fazendo com que elas participem de forma efetiva das decisões sobre sua saúde. Para tanto, o profissional de enfermagem não deve deixar de prestar uma assistência humanizada, visualizando os aspectos biopsicossociais, que possibilitam uma assistência efetiva e de qualidade, pois através de um olhar holístico o enfermeiro obtém mais subsídios para realizar intervenções eficientes com o objetivo de sanar as dúvidas das adolescentes (NETTO JJ, et al, 2017).

Nessa perspectiva de cuidado centrado na adolescente, esse grupo específico muitas vezes estão imerso em alguns quadros clínicos que merecem total investigação e construção de uma linha de cuidados compartilhada entre a equipe. Uma pesquisa, identificou os principais diagnósticos de uma consulta de medicina. Os resultados revelaram que, o grupo das doenças endócrinas, nutricionais e metabólicas foi o mais representativo da amostra (34\%), sendo a obesidade o diagnóstico mais frequente em ambos os sexos e grupos etários (23\%), com maior prevalência nos rapazes e nos adolescentes mais jovens. O grupo dos transtornos mentais e do comportamento foi o segundo mais prevalente (32\%), afetando principalmente as adolescentes e o grupo etário mais velho. Como também, os problemas sociais constituíram o principal diagnóstico em $8 \%$ das consultas (ABREU N, et al, 2018).

Durante o processo da coleta dos dados, foi possível perceber a dificuldade de trazer as adolescentes para a unidade, existindo uma forte resistência à adesão a atividades realizadas para elas. Contudo, a partir do momento que elas compareciam e se disponibilizaram a participar da consulta o cenário era outro, imediatamente surgiam sentimentos positivos acerca do atendimento de Enfermagem específico e individualizado, percebe-se, portanto, que essas passam a sentir-se pertencentes do serviço. De acordo com o manual "Proteger e cuidar da saúde dos adolescentes na atenção básica", é imprescindível que os Enfermeiros (as) percebam que as ações voltadas para as adolescentes precisam ser planejadas de modo que respeitem a privacidade de cada uma, sejam essas coletivas ou individuais (BRASIL, 2017).

Desse modo, a consulta de Enfermagem deve ser baseada em conhecimento científico, fundamentandoa em um modelo teórico de Enfermagem com vistas a determinar as necessidades e o grau de dependência 
dos indivíduos, família e/ou comunidade (DOMINGOS CS, et al, 2015). Ressaltando que, a experiência na elaboração e utilização dos instrumentos na consulta de Enfermagem é eficiente quanto à forma e ao conteúdo, uma vez que esses possibilitam um olhar ampliado do processo saúde-doença, além de facilitar a atuação do enfermeiro na abordagem integral do paciente (TRINDADE LR, et al, 2016).

\section{A criação do vínculo de confiança através da consulta}

A consulta de enfermagem permitiu criar um vínculo de confiança entre o enfermeiro e a adolescente.

"Eu achei bom, porque às vezes é bom a gente [...] ter alguém pra conversar, porque eu pensei que ia ser uma coisa bem assim, "ai meu Deus do céu, que vergonha", mas não foi não. Foi bem legal, eu gostei muito [...]. " (A2)

"Foi bom. É assim, como não tem nada relacionado à, a adolescente no posto aqui nem nada, é uma forma de interesse também, porque tipo assim, coisas que eu conversei com ela, é, eu, eu não converso com a minha mãe, tipo com ninguém do meu ciclo social." (A4)

"Porque assim, eu nunca tinha se aberto, assim, tanto com uma pessoa... eu tenho muita vergonha, então pra eu me abrir assim com uma pessoa é bem difícil, sempre tenho muita vergonha. Mas dessa vez foi diferente, eu não fiquei assim, com muita vergonha, entendeu?" (A6)

"Não me senti constrangida, gostei, porque tava querendo saber mais da gente. No começo eu tive [...], tava agoniada, mas depois eu fiquei relaxada." (A8)

"Foi legal! Porque me ajudou em algumas coisas! Por exemplo, é, o negócio da menstruação, que eu tava em dúvida, e eu tenho vergonha de tirar essas dúvidas com mainha! [...] Hum, fiquei um pouquinho nervosa, porque, eu nunca fiz uma consulta assim de Enfermagem." (A10)

Nota-se que as adolescentes entrevistadas se sentiram à vontade para conversar sobre situações íntimas que nem mesmo conseguiam expressar com seus familiares mais próximos. Com base nessa perspectiva, os serviços de saúde devem articular ações que facilitem o engajamento do jovem adolescente em seu cuidado em diferentes dimensões. Através da fácil acessibilidade e do acolhimento com os profissionais, os adolescentes devem estabelecer suas relações de vínculo com os profissionais de saúde e, desse modo, possibilitar a construção da autonomia para compartilhar a tomada de decisão sobre as possibilidades de preservar a saúde (COSTA RF, et al, 2012).

É de salutar importância que diante dessa rede de apoio, os jovens se sintam aceitos, confortados, ajudados e respeitados no que se refere aos seus direitos de adolescentes para que possam confiarem na equipe e possibilitar o retorno aos serviços de saúde quando necessário. Dessa forma, o modo em que o adolescente é recebido nas unidades irá definir o vínculo necessário para a continuidade do cuidado (COSTA RF, et al, 2012).

Um estudo sobre o cuidado ao adolescente na atenção primária mostrou que uma pequena quantidade de adolescentes fazia parte do grupo dos usuários em geral que participavam dor serviços de saúde. Assim, os autores pontuam que é necessário a construção de um plano de ação baseado fortemente no acolhimento e vínculo, de uma equipe multiprofissional, materiais e insumos próprios para atender a essa população específica. (QUEIROZ MVO, et al, 2011).

Desse modo, as práticas assistenciais de saúde devem ser embasadas na responsabilização clínica e da capacidade intervencionista resolutiva por parte dos profissionais, devendo ser resolutivo e servir como um facilitador desse processo de cuidado contínuo, como forma de atingir a integralidade da atenção e autonomia do adolescente (COSTA RF, et al, 2012).

\section{Falta de atividades educativas em saúde na UBS}

De acordo com o modelo tradicional que permeia a nossa sociedade, o adolescente é esquecido quando se trata do reconhecimento da sua singularidade e especificidades pertencentes a esta fase da juventude e 
passa a ser notado como um usuário comum que recorre ao serviço de saúde. No mais, no modelo atual de atenção, a equipe de saúde estabelece uma relação vertical com o adolescente, impondo-Ihe normas de conduta, acreditando que, assim fazendo, proporciona ao usuário qualidade no atendimento. Essa postura coloca o adolescente em uma posição de inferioridade e de passividade, retirando dele não só a liberdade de escolha, como também a responsabilidade por seus atos (SANTOS CC e RESSEL LB, 2013).

Nesse cenário desafiador, é notório uma carência de atividades e de ações internas e externas no contexto da Atenção Básica para o público alvo das adolescentes. Esse déficit é apontado na seguinte fala:

"Como falei, mal tem coisa de adolescente aqui, e, eles mal fazem essas coisas... porque tipo quando a pessoa chega aqui eles, entre aspas, mal atendem." (A7)

Nesse contexto desafiador, a discussão acerca da saúde do adolescente ainda é incipiente, o que pode ser reflexo da política nacional de saúde que, durante décadas, priorizou, em suas diretrizes gerais, a assistência ao grupo materno infantil (NOGUEIRA MJ et al, 2010). Observa-se, então, fragilidade nas ações voltadas à saúde do adolescente, em detrimento às ações da saúde da criança, adulto e idoso (SILVA JM e CALDEIRA AP, 2010).

Estudo identificou que os profissionais atendem adolescentes, diariamente ou quase todos os dias, no entanto, quanto ao motivo da procura por atendimento médico, evidenciou-se que eles os procuram para tratamento de doenças, mostrando que o motivo da procura pelo serviço tem estado bastante ligado a problemas clínicos (FERRARI RAP, et al, 2008). Como também, em outro estudo realizado com profissionais da ESF, em Florianópolis, constatou que a formação dos profissionais de saúde é uma das problemáticas centrais, no contexto investigado, em se trabalhar a educação em saúde na lógica da promoção da saúde requerida pela ESF (BESEN CB, et al, 2013).

Reconhece-se a importância das ações de educação em saúde nas escolas. No processo de busca da promoção da saúde, utilizam-se as estratégias de educação em saúde como uma ferramenta para transformar os usuários em sujeitos autônomos e participativos do processo de saúde (MACHADO MFAS, 2010). Ressaltando o Programa Saúde na Escola - PSE, instituído pelo Decreto Presidencial no 6.286/2007, que surgiu como uma política intersetorial entre os Ministérios da Saúde e da Educação, com a finalidade de prestar atenção integral (prevenção, promoção e atenção) à saúde de crianças, adolescentes e jovens do ensino público básico, no âmbito das escolas e unidades básicas de saúde, realizadas pelas Equipes de Saúde e educação de forma integrada (BRASIL, 2010).

\section{CONCLUSÃO}

Conclui-se que para as adolescentes, a consulta de enfermagem na atenção básica representa um meio de esclarecer dúvidas sobre saúde, além de promover um vínculo de confiança da adolescente com o enfermeiro. Em seus relatos, ficou enfatizada a importância da consulta de enfermagem, por possibilitar esclarecer dúvidas, questionamentos e conversar sobre assuntos considerados íntimos. Desta forma, a consulta de enfermagem ao adolescente se apresenta como uma tecnologia leve de intervenção da realidade, por meio da promoção da escuta e diálogo, tendo a própria adolescente como protagonista nas suas escolhas que influenciam o processo de saúde-doença. Contudo, também há o desejo das adolescentes de ter atividades e atendimentos voltados para elas na $A B$, uma vez que houve dificuldade em trazer esse público para participar da consulta de Enfermagem.

\section{REFERÊNCIAS}

1. ALENCAR RA, et al. Desenvolvimento de uma proposta de educação sexual para adolescentes. Revista Ciência e Educação. 2008; 14(01): 159-168.

2. ABREU N, et al. Quais os diagnósticos mais frequentes na adolescência? A realidade de uma consulta de Medicina do Adolescente. einstein. 2018;16(2):eAO4225. 
3. BRASIL, Ministério da Saúde. Secretaria de Atenção à Saúde. Departamento de Ações Programáticas e Estratégicas. Proteger e cuidar da saúde de adolescentes na atenção básica [recurso eletrônico] Brasília: Ministério da Saúde, 2017.

4. BRASIL, Ministério da Saúde. Secretária de Atenção em Saúde. Departamento de Ações Programáticas Estratégicas. Diretrizes nacionais para a atenção integral à saúde de adolescentes e jovens na promoção, proteção e recuperação da saúde. Área técnica de Saúde do Adolescente e do Jovem. Brasília: Ministério da Saúde, 2010.

5. BRASIL, Conselho Nacional de Saúde. Resolução n510, de 07 de abril de 2016. Aprova normas regulamentadoras de pesquisas envolvendo seres humanos. Brasília: Diário Oficial da União, 2016.

6. BRASIL, Ministério da Saúde. Portaria n 2.488, de 21 de outubro de 2011. Aprova a Política Nacional de Atenção Básica, estabelecendo a revisão de diretrizes e normas para a organização da Atenção Básica, para a Estratégia Saúde da Família (ESF) e o Programa de Agentes Comunitários de Saúde (PACS). Brasília; 2011.

7. BRASIL. Departamento de Atenção Básica. Programa Saúde na Escola. 2010.

8. BESEN CB, et al. A estratégia saúde da família como objeto de educação em saúde. Saude Soc. 2017;16(1):57-68.

9. COSTA RF, et al. Cuidado aos adolescentes na Atenção Primária: perspectivas de integralidade. Esc Anna Nery. 2012; 16 (3):466-4722.

10. DOMINGOS CS, et al. Construction and validation of the historical contents of nursing guided by orem reference. REME rev min enferm. 2015;19(2):165-75.

11. FERRARI RAP, et al. Adolescência: ações e percepção dos médicos e enfermeiros do Programa Saúde da Família. Interface - Comunic, Saúde, Educ. 2008;12(25):387-400.

12. FERRARI RAP, et al. Atenção a saúde dos adolescentes: percepção dos médicos e enfermeiros das equipes da saúde da família. Cad. Saúde Pública. 2006; 22(11): 2491-2495.

13. MACHADO MFAS, et al. Compreensão das mudanças comportamentais do usuário no Programa Saúde da Família por meio da participação habilitadora. Ciênc Saúde Coletiva. 2010;15(4):2133-43.

14. NOGUEIRA MJ et al. Políticas públicas voltadas para adolescentes nas unidades básicas de saúde no município de Belo Horizonte/MG: uma análise sob a perspectiva dos profissionais de saúde. Rev APS. 2010;13(3):338-45.

15. NETTO JJ, et al. Atenção à saúde do adolescente na estratégia de saúde da família: do individual ao grupal. Revista Adolescência e Saúde. 2017; 14(2):189-193.

16. QUEIROZ, et al. Cuidado ao adolescente na atenção primária: discurso dos profissionais sobre o enfoque da integralidade. Rev RENE. 2011; 12: 1036-44.

17. SANTOS CC e RESSEL LB. O adolescente no serviço de saúde. Adolesc. Saude. 2013; 10 (1): 53-55.

18. SILVA JM, Caldeira AP. Modelo assistencial e indicadores de qualidade da assistência: percepção dos profissionais da atenção primária à saúde Cad Saúde Pública. 2010; 26(6):1187-93.

19. TRINDADE LR, et al. Nursing process: challenges and strategies for its implementation from the nurses' point of view. Saúde (Santa Maria). 2016;42(1):75-82.

20. VIEIRA RP, et al. Participação de adolescentes na estratégia de saúde da família a partir da estrutura teóricometodologica de uma participação habilitadora. Rev Latino-Am Enfermagem 2014; 22(2): 309-316. 